\title{
Chaotic and Hyperchaotic Complex Jerk Equations
}

\author{
Gamal M. Mahmoud, Mansour E. Ahmed \\ Department of Mathematics, Faculty of Science, Assiut University, Assiut, Egypt \\ Email: gmahmoud@aun.edu.eg
}

Received January 27, 2012; revised February 29, 2012; accepted March 12, 2012

\begin{abstract}
The aim of this paper is to introduce and investigate chaotic and hyperchaotic complex jerk equations. The jerk equations describe various phenomena in engineering and physics, for example, electrical circuits, laser physics, mechanical oscillators, damped harmonic oscillators, and biological systems. Properties of these systems are studied and their Lyapunov exponents are calculated. The dynamics of these systems is rich in wide range of systems parameters. The control of chaotic attractors of the complex jerk equation is investigated. The Lyapunov exponents are calculated to show that the chaotic behavior is converted to regular behavior.
\end{abstract}

Keywords: Hyperchaotic; Chaotic; Attractors; Lyapunov Exponents; Jerk Function; Control; Complex

\section{Introduction}

Chaos and hyperchaos can occur in systems of autonomous ordinary differential equations (ODE's) with at least three variables and two quadratic nonlinearities [1-4]. The Poincaré-Bendixson theorem shows that chaos does not exist in a two-dimensional autonomous system. In 1994, Sprott [5] found numerically fourteen chaotic systems with six terms and one quadratic nonlinearities. In 1996, Gottlieb [6] showed that some of these systems could be written as a single third-order ODE. By "jerk function" he means a function $j$ such that the third-order ODE can be written in the form $\dddot{\theta}=j(\theta, \dot{\theta}, \ddot{\theta})$, where $j$ is the time derivative of the acceleration $\ddot{\theta}$ and the equation is called a jerk equation. The jerk equations describe various phenomena in engineering and physics, for example, electrical circuits, laser physics, mechanical oscillators, damped harmonic oscillators, and biological systems. In the literature some jerk equations are introduced and studied [7-13]. Kocić et al. [14] considered and studied two modifications of a 3-dimensional dynamic flow known as jerk dynamical systems of Sprott [13].

In this paper, we define a complex jerk equation as an autonomous third-order complex differential equation of the form:

$$
\dddot{z}=j(z, \bar{z}, \dot{z}, \dot{\bar{z}}, \ddot{z}, \ddot{\bar{z}}),
$$

where $z$ is a complex variable, the overdot represent the time derivative and $j$ is the complex jerk function (time derivative of acceleration $\ddot{z}$ ) and an overbar denotes complex conjugate variables. In recent years we introduced and studied several complex nonlinear systems which appear in many important applications [15-21].

As an example of Equation (1), we propose a chaotic complex jerk equation as:

$$
\dddot{z}+\alpha \ddot{z}+\beta \dot{z}+v z+\eta z^{2} \bar{z}=0
$$

where $\alpha, \beta$ and $\eta$, are positive parameters, $v$ is a negative parameter, $z$ is complex variable, dots represent derivatives with respect to time and an overbar denotes complex conjugate variables.

We suggest the equation:

$$
\dddot{z}+\alpha \ddot{z}-z \dot{z}^{2}+z=0, \alpha>0,
$$

which is an example of a hyperchaotic complex jerk equation. The corresponding real form of (3) (i.e. $z$ is a real variable) was introduced in [9], and has only chaotic behavior.

The organization of rest of the paper is as follows: In Section 2, symmetry, invariance, fixed points of (2) and stability analysis of the trivial fixed point are discussed. Lyapunov exponents are calculated in Section 3, and used to classify the attractors of (2). It is clear that our Equation (2) has chaotic, periodic, quasi-periodic attracttors and solutions that approach fixed points. In Section 4, we study the basic properties of the hyperchaotic complex jerk Equation (3). Numerically the range of pa- rameters values of the system at which hyperchaotic attractors exist is calculated in Section 5. Section 6 contains the control of chaotic attractors of Equation (2) by adding a complex periodic forcing. The last section contains our concluding remarks.

\section{Basic Properties of Chaotic Jerk Equation (2)}

In this section we study the basic properties of our new Equation (2). Equation (2) can equivalently be written as three, first-order, ordinary differential equations as: 


$$
\dot{z}=x, \dot{x}=y, \dot{y}=-\alpha y-\beta x-v z-\eta z^{2} \bar{z},
$$

where $z=u_{1}+i u_{2}, \quad x=u_{3}+i u_{4}$ and $y=u_{5}+i u_{6}$ are complex variables, $i=\sqrt{-1}, u_{j}, j=1,2, \cdots, 6$ are real variables, dots represent derivatives with respect to time and an overbar denotes complex conjugate variables.

The real version of (4) is:

$$
\begin{aligned}
& \dot{u}_{1}=u_{3}, \dot{u}_{2}=u_{4}, \dot{u}_{3}=u_{5}, \dot{u}_{4}=u_{6}, \\
& \dot{u}_{5}=-\alpha u_{5}-\beta u_{3}-v u_{1}-\eta u_{1}\left(u_{1}^{2}+u_{2}^{2}\right), \\
& \dot{u}_{6}=-\alpha u_{6}-\beta u_{4}-v u_{2}-\eta u_{2}\left(u_{1}^{2}+u_{2}^{2}\right) .
\end{aligned}
$$

The basic dynamical properties of system (5) are:

\subsection{Symmetry and Invariance}

From (5), we note that this system is invariant under the transformation $\left(u_{1}, u_{2}, u_{3}, u_{4}, u_{5}, u_{6}\right) \Leftrightarrow\left(-u_{1},-u_{2},-u_{3},-u_{4},-u_{5},-u_{6}\right)$.

Therefore, if $\left(u_{1}, u_{2}, u_{3}, u_{4}, u_{5}, u_{6}\right)$ is a solution of (5), then $\left(-u_{1},-u_{2},-u_{3},-u_{4},-u_{5},-u_{6}\right)$ is also a solution of the same system.

\subsection{Dissipation}

The divergence of (5) is: $\sum_{j=1}^{6} \frac{\partial \dot{u}_{j}}{\partial u_{j}}=-2 \alpha$.

Therefore the system (5) is dissipative for the case: $\alpha>0$.

\subsection{Equilibria and Their Stability}

The fixed points of system (5) can be found by solving the following equations:

$$
\begin{aligned}
& u_{3}=0, u_{4}=0, u_{5}=0, u_{6}=0, \\
& -\alpha u_{5}-\beta u_{3}-v u_{1}-\eta u_{1}\left(u_{1}^{2}+u_{2}^{2}\right)=0, \\
& -\alpha u_{6}-\beta u_{4}-v u_{2}-\eta u_{2}\left(u_{1}^{2}+u_{2}^{2}\right)=0 .
\end{aligned}
$$

Therefor system (4) has trivial fixed point $E_{0}=(0$, $0,0,0,0,0)$. The projection in the $\left(u_{1}, u_{2}\right)$ plane of the non trivial fixed points is a circle:

$$
u_{1}^{2}+u_{2}^{2}=r^{2},
$$

whose center is at the origin and radius is $r=\sqrt{-v / \eta}$.

The non trivial fixed point can be written in the form

$$
\begin{aligned}
& E_{\theta}=\left(u_{1}, u_{2}, u_{3}, u_{4}, u_{5}, u_{6}\right) \text {, } \\
& L_{l, j}=\left(\begin{array}{cccccc}
0 & 0 & 1 & 0 & 0 & 0 \\
0 & 0 & 0 & 1 & 0 & 0 \\
0 & 0 & 0 & 0 & 1 & 0 \\
0 & 0 & 0 & 0 & 0 & 1 \\
-v-\eta\left(3 u_{1}^{2}+u_{2}^{2}\right) & -2 \eta u_{1} u_{2} & -\beta & 0 & -\alpha & 0 \\
-2 \eta u_{1} u_{2} & -v-\eta\left(3 u_{2}^{2}+u_{1}^{2}\right) & 0 & -\beta & 0 & -\alpha
\end{array}\right) .
\end{aligned}
$$
for $\theta=[0,2 \pi]$. Equation (2) at $E_{0}$ is:

The characteristic polynomial is:

Since, $v<0$ then $E_{0}$ is unstable. as we did for the trivial fixed point $E_{0}$. fixed point exist.

\subsection{Lyapunov Exponents} $U(t)$ are:

where $u_{1}=r \cos \theta, \quad u_{2}=r \sin \theta, \quad u_{3}=u_{4}=u_{5}=u_{6}=0$,

To study the stability of $E_{0}$ the Jacobian matrix of

$$
J_{E_{0}}=\left(\begin{array}{cccccc}
0 & 0 & 1 & 0 & 0 & 0 \\
0 & 0 & 0 & 1 & 0 & 0 \\
0 & 0 & 0 & 0 & 1 & 0 \\
0 & 0 & 0 & 0 & 0 & 1 \\
-v & 0 & -\beta & 0 & -\alpha & 0 \\
0 & -v & 0 & -\beta & 0 & -\alpha
\end{array}\right) .
$$

$$
\left(\lambda^{3}+\alpha \lambda^{2}+\beta \lambda+v\right)^{2}=0
$$

According to the Routh-Hurwitz condition, the real parts of the roots $\lambda$ of (8) are negative if and only if

$$
\alpha>0, \beta>0, v>0, \alpha \beta-v>0 .
$$

The stability analysis of $E_{\theta}$ can be similarly studied

\section{Lyapunov Exponents and Attractors of Equation (2)}

This section is devoted to calculate Lyapunov exponents and used their signs to classify attractors of Equation (2). Based on these exponents, we compute parameters values of our Equation (2) at which chaotic, periodic, and quasi-periodic attractors and attractors that approach

System (5) in vector notation can be written as:

$$
\dot{U}(t)=h(U(t) ; \eta),
$$

where $U(t)=\left[u_{1}(t), u_{2}(t), u_{3}(t), u_{4}(t), u_{5}(t), u_{6}(t)\right]^{t}$ is the state space vector, $h=\left[h_{1}, h_{2}, h_{3}, h_{4}, h_{5}, h_{6}\right]^{t}, \quad \eta$ is a set of parameters and $[\cdots]^{t}$ denoting transpose. The equations for small deviations $\delta U$ from the trajectory

$$
\delta \dot{U}(t)=L_{l j}(U(t) ; \eta) \delta U, \quad l, j=1,2,3,4,5,6
$$

where $L_{l, j}=\frac{\partial h_{l}}{\partial u_{j}}$ is the Jacobian matrix of the form: 
The Lyapunov exponents $\lambda_{l}$ of the system is defined by:

$$
\lambda_{l}=\lim _{t \rightarrow \infty} \frac{1}{t} \log \frac{\left\|\delta u_{l}(t)\right\|}{\left\|\delta u_{l}(0)\right\|} .
$$

To find $\lambda_{l}$, Equations (9) and (10) must be numerically solved simultaneously. Runge-Kutta method of order 4 is used to calculate $\lambda_{1}$.

For the choice $\alpha=1, \beta=4, v=-5$ and $\eta=1$ and the initial conditions $t_{0}=0, u_{1}(0)=4, u_{2}(0)=1, u_{3}(0)=-2$, $u_{4}(0)=2, u_{5}(0)=-1$ and $u_{6}(0)=1$. We calculate the Lyapunov exponents which are: $\lambda_{1}=0.244, \lambda_{2}=0, \lambda_{3}=0$, $\lambda_{4}=-0.488, \lambda_{5}=-0.954, \lambda_{6}=-1.691$.

This means that our system (4) for this choice of $\alpha, \beta$, $\mu$ and $\eta$ is a chaotic system since one of its Lyapunov exponents $\lambda_{1}$ is positive and dissipative system because their sum is negative.

\subsection{Attractors of Equation (2)}

\subsubsection{Fix $\beta=4, \quad v=-5, \quad \eta=1$ and Vary $\alpha$}

In Figures 1(a) and (b) we plotted the corresponding Lyapunov exponents $\lambda_{l}, l=1,2, \cdots, 6$ of system (5) using the initial conditions $t_{0}=0, u_{1}(0)=4, u_{2}(0)=1$, $u_{3}(0)=-2, \quad u_{4}(0)=2, \quad u_{5}(0)=-1$ and $u_{6}(0)=1$. It is clear that from Figure 1(a), when $\alpha \in[0.65,0.795]$, $(0.840,1.025]$ and $(1.26,1.34]$ the new system (5) has chaotic attractors. It has also periodic attractors for $\alpha \in(1.34,2.54]$, while it has quasi-periodic attractors when $\alpha$ lies in the interval $(0.795,0.840]$. Solutions of system (5) that approach nontrivial fixed points are exist for $\alpha \in(2.54,4.7)$. As is shown in Figure 1(b) the values of $\lambda_{4}, \lambda_{5}$ and $\lambda_{6}$ are negative.

3.2.2. Fix $\alpha=1, \quad v=-5, \quad \eta=1$ and Vary $\beta$ From Figure 1(c) one can conclude that (5) has chaotic attractors for $\beta \in[3.4,3.590],[3.610,3.925],[3.955$, $4.135]$ and $(4.155,4.880]$, and periodic attractors for $\beta$ lies in $(3.590,3.610],[4.135,4.155]$ and $(4.880,9.8]$. Solutions of system (5) that approach nontrivial fixed points are exist for $\beta \in(10.1,12.4)$.

\subsubsection{Fix $\alpha=1, \quad \beta=4, \quad \eta=1$ and Vary $v$}

As we did before we plot only $\lambda_{1}, \lambda_{2}$ and $\lambda_{3}$ in Figure 1(d) and we see that (5) has chaotic attractors for $v \in[-6.7,-6.315],(-6.240,-5.955],(-5.865,-5.580]$, $(-5.525,-5.105],(-5.07,-4.805]$ and

$(-4.775,-3.995]$. In between above values of $v$ our system has periodic attractors as one sees from Figure 1(d).

3.2.4. Fix $\alpha=1, \quad \beta=4, \quad v=-5$ and Vary $\eta$ As is shown in Figure 1(e), the chaotic attractors exist for $\eta \in[0.001,1.14]$.

Using the same choice of the parameters and initial conditions as in Figure 1, the chaotic attractors of (5) are plotted in Figure 2 in $\left(u_{2}, u_{3}, u_{4}\right),\left(u_{2}, u_{4}, u_{6}\right),\left(u_{2}\right.$, $\left.u_{4}, u_{5}\right)$ and $\left(u_{1}, u_{4}, u_{5}\right)$ spaces respectively.

\section{Some Properties of Equation (3)}

This section deals with the basic dynamical properties of a hyperchaotic jerk Equation (3). As we did in Section 2, Equation (3) can be written as a system of three, firstorder, ordinary differential equations such as:

$$
\dot{z}=x, \dot{x}=y, \dot{y}=-\alpha y-z x^{2}-z,
$$

where $z=u_{1}+i u_{2}, x=u_{3}+i u_{4}$ and $y=u_{5}+i u_{6}$ are complex variables, $i=\sqrt{-1}, u_{j}, j=1,2, \cdots, 6$ are real variables.

The real version of (3) is:

$$
\begin{aligned}
& \dot{u}_{1}=u_{3}, \dot{u}_{2}=u_{4}, \dot{u}_{3}=u_{5}, \dot{u}_{4}=u_{6}, \\
& \dot{u}_{5}=-\alpha u_{5}+u_{1}\left(u_{3}^{2}-u_{4}^{2}\right)-2 u_{2} u_{3} u_{4}-u_{1}, \\
& \dot{u}_{6}=-\alpha u_{6}+u_{2}\left(u_{3}^{2}-u_{4}^{2}\right)+2 u_{1} u_{3} u_{4}-u_{2} .
\end{aligned}
$$

If $\left(u_{1}, u_{2}, u_{3}, u_{4}, u_{5}, u_{6}\right)$ is a solution of (13), then $\left(-u_{1}\right.$, $\left.-u_{2},-u_{3},-u_{4},-u_{5},-u_{6}\right)$ is also a solution.

From (13) if $\alpha>0$, then (13) is dissipative.

System (13) has only trivial fixed point $E_{0}=(0,0$, $0,0,0,0)$.

To study the stability of $E_{0}$ we calculate the Jacobian matrix of system (13) at $E_{0}$ to get:

$$
J_{E_{0}}=\left(\begin{array}{cccccc}
0 & 0 & 1 & 0 & 0 & 0 \\
0 & 0 & 0 & 1 & 0 & 0 \\
0 & 0 & 0 & 0 & 1 & 0 \\
0 & 0 & 0 & 0 & 0 & 1 \\
-1 & 0 & 0 & 0 & -\alpha & 0 \\
0 & -1 & 0 & 0 & 0 & -\alpha
\end{array}\right) .
$$

Its characteristic polynomial is:

$$
P(\lambda)=\left(\lambda^{3}+\alpha \lambda^{2}+1\right)^{2}=0 .
$$

An elementary study proves that this polynomial has only one real root $\lambda_{r}<\frac{-2 \alpha}{3}$ which is therefore negative. Since the characteristic equation is a cubic equation with real coefficients, we will have without loss of generality $P(\lambda)=\left(\lambda-\lambda_{r}\right)\left(\lambda-\lambda_{c}\right)\left(\lambda-\bar{\lambda}_{c}\right)$. where $\lambda_{c}$ is a complex number. After expanding the above equation and comparing the coefficients with those of the original characteristic equation we come up with the relation $\operatorname{Re}\left(\lambda_{c}\right)=2^{-1} \lambda_{r}^{-2}$. We conclude that the real part of $\lambda_{c}$ is positive and that the fixed point is unstable, see Ref. [9].

\section{Lyapunov Exponents and Attractors of Hyperchaotic Equation (3)}

In this section we calculate the Lyapunov exponents and attractors of Equation (3). 


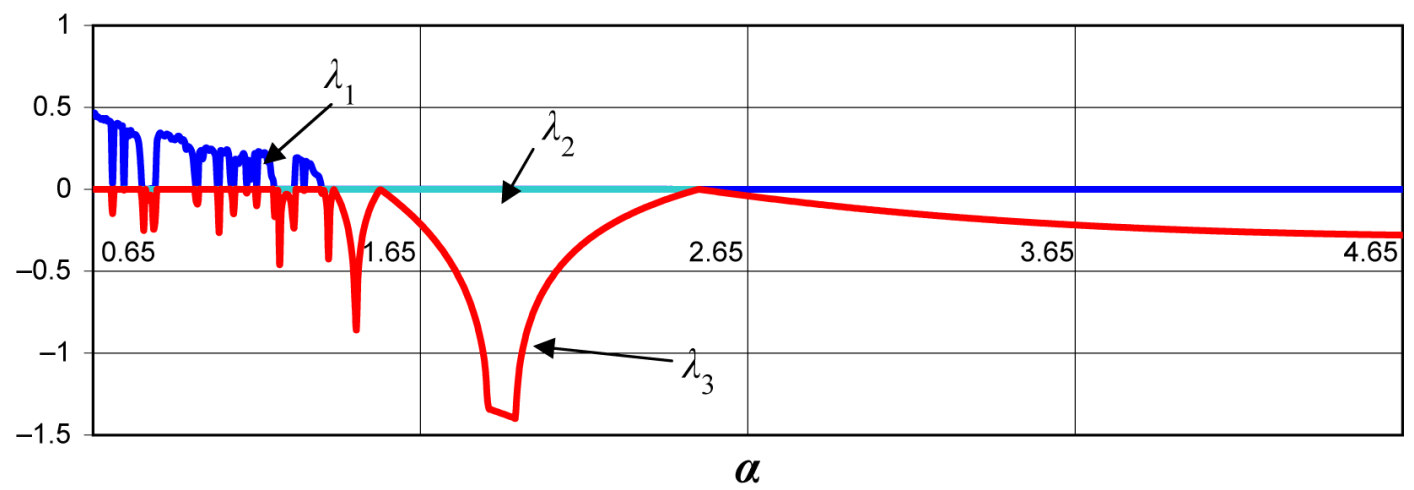

(a)

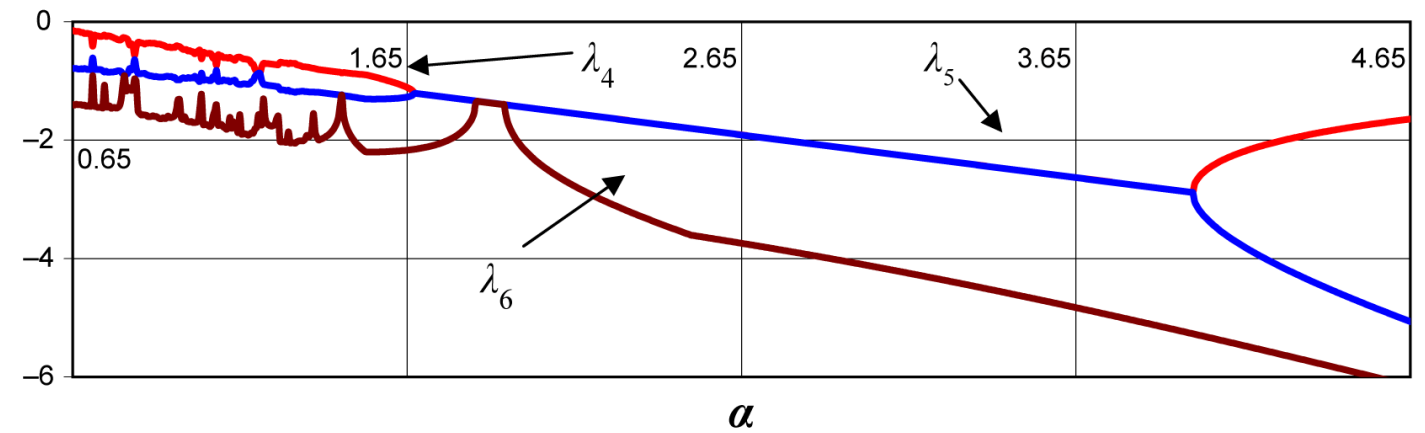

(b)

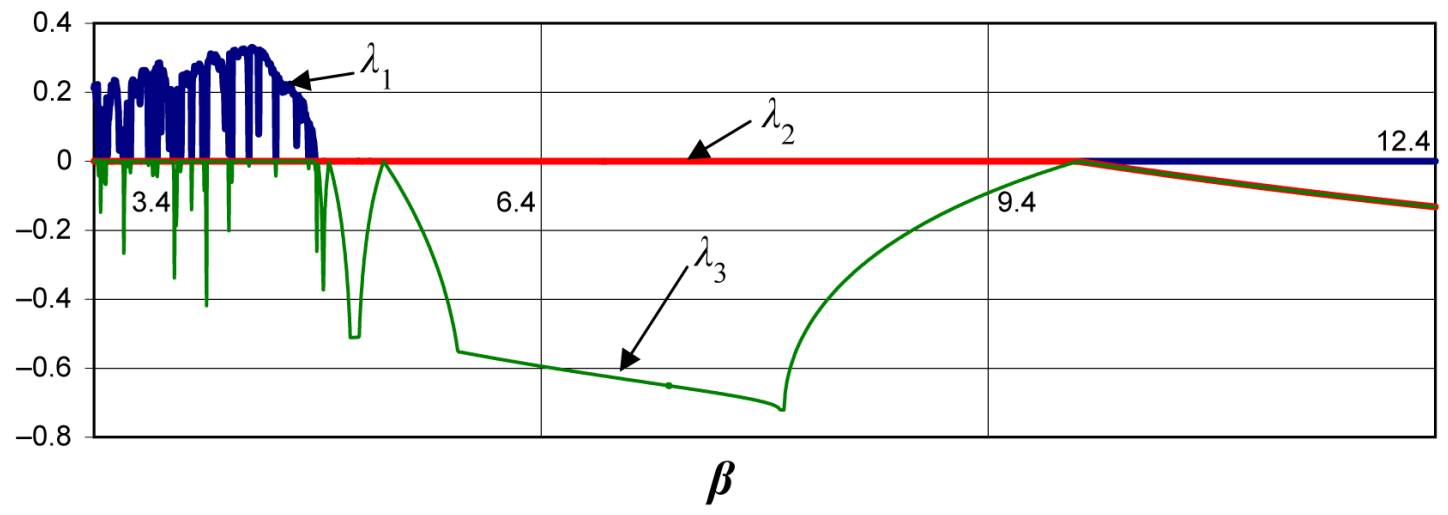

(c)

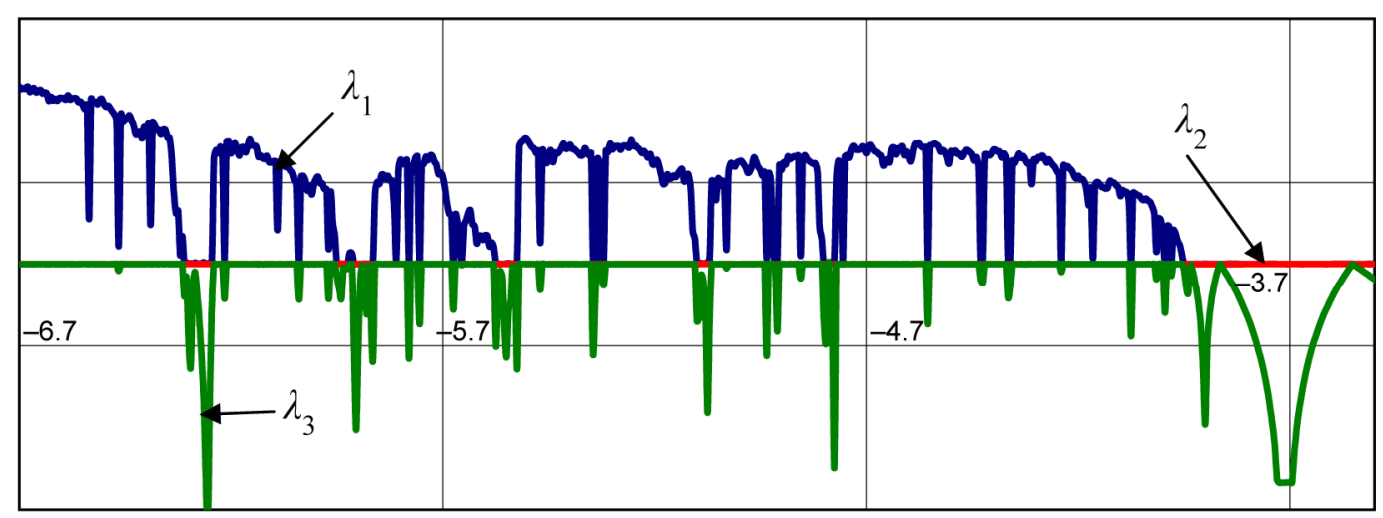

0.6

0.2 


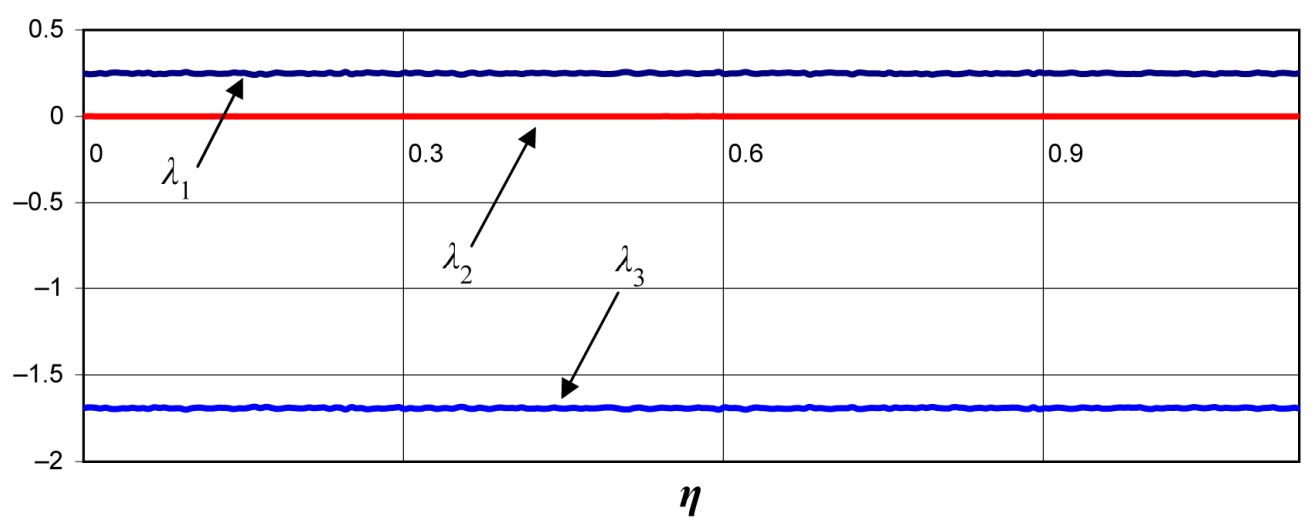

(e)

Figure 1. Lyapunov exponents of (2) with the initial conditions $t_{0}=0, \quad u_{1}(0)=4, u_{2}(0)=1, \quad u_{3}(0)=-2, u_{4}(0)=2$, $u_{5}(0)=-1$ and $u_{6}(0)=1$. (a) $\lambda_{1}, \lambda_{2}$ and $\lambda_{3}$ versus $\alpha$; (b) $\lambda_{4}, \lambda_{5}$ and $\lambda_{6}$ versus $\alpha$; (c) $\lambda_{1}, \lambda_{2}$ and $\lambda_{3}$ versus $\beta$; (d) $\lambda_{1}, \lambda_{2}$ and $\lambda_{3}$ versus $v$; (e) $\lambda_{1}, \lambda_{2}$ and $\lambda_{3}$ versus $\eta$.

(a)

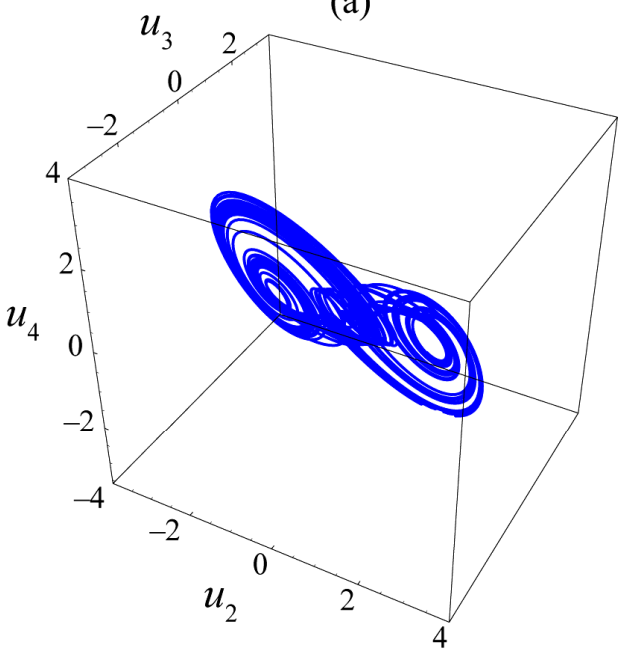

(c)

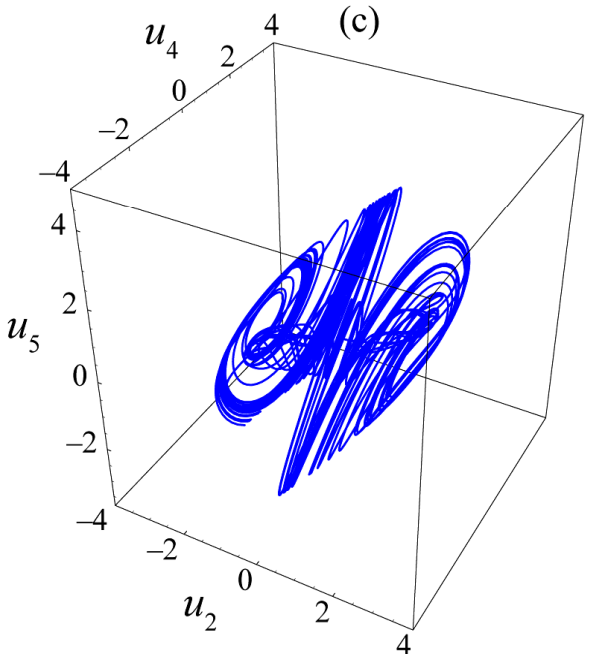

(b)

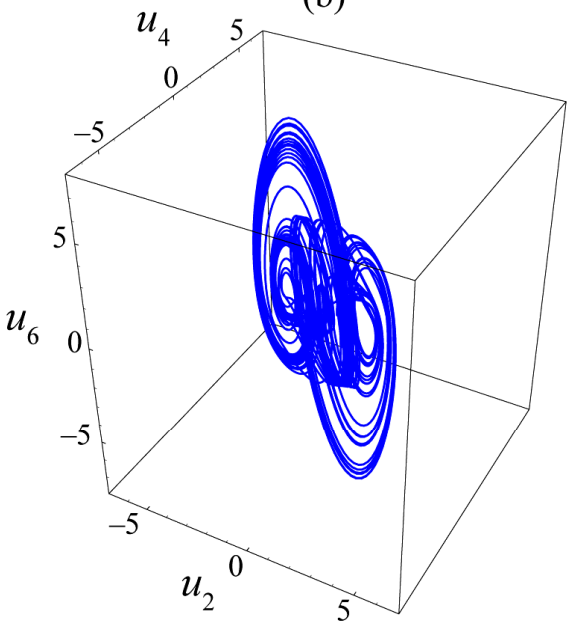

(d)

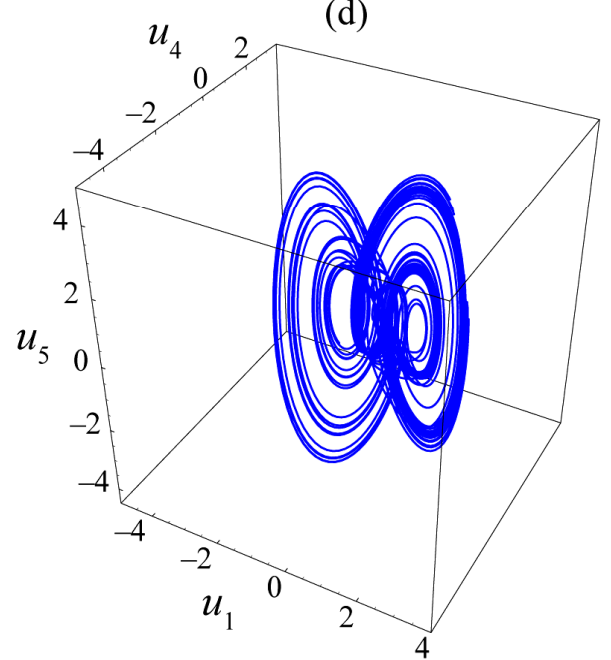

Figure 2. A chaotic attractor of (2) for $\alpha=1, \beta=4, v=-5$ and $\eta=1$ at the same initial conditions as in Figure 1. (a) in $\left(u_{2}, u_{3}, u_{4}\right)$ space; (b) in $\left(u_{2}, u_{4}, u_{6}\right)$ space; (c) in $\left(u_{2}, u_{4}, u_{5}\right)$ space; (d) in $\left(u_{1}, u_{4}, u_{5}\right)$ space. 
For the choice $\alpha=2.03$ and the initial conditions $t_{0}=0, \quad u_{1}(0)=4, \quad u_{2}(0)=0, \quad u_{3}(0)=0, \quad u_{4}(0)=0$, $u_{5}(0)=-1$ and $u_{6}(0)=0$, we calculate the Lyapunov exponents which are: $\lambda_{1}=0.111018, \lambda_{2}=0.111018$, $\lambda_{3}=0, \lambda_{4}=0, \lambda_{5}=-3.03968$ and $\lambda_{6}=-3.03968$. This means that our Equation (3) for this choice of $\alpha$ is hyper chaotic equation since two of the Lyapunov exponents $\lambda_{1}$ and $\lambda_{2}$ are positive and dissipative equation since the sum of its Lyapunov exponents is negative.

In Figure 3(a) we plotted the corresponding Lyapunov exponents $\lambda_{l}, \quad l=1,2, \cdots, 6$ of Equation (3) using the initial conditions $t_{0}=0, u_{1}(0)=4, u_{2}(0)=0, u_{3}(0)=0$, $u_{4}(0)=0, u_{5}(0)=-1$ and $u_{6}(0)=0$. It is clear that from Figure 3(a), when $\alpha \in(2.0278,2.0413],(2.0430,2.0539]$, $(2.0544,2.0732]$ and $(2.0743,2.0840]$ Equation (3) has hyperchaotic attractors, while it has quasi-periodic attracttors when $\alpha$ lies in the intervals, $(2.0413,2.0430$ ], $(2.0732,2.0743]$ and $(2.0840,2.1]$.

The hyperchaotic attractors of (3) using the same initial conditions as in Figure 3(a) and for $\alpha=2.03$, are plotted in Figures 3(b) and (c) in $\left(u_{2}, u_{3}, u_{5}\right)$ and $\left(u_{1}, u_{2}, u_{3}\right)$ spaces respectively.

\section{Control of Chaotic Attractors of System (4)}

This section is devoted to study the control of chaotic attractors of system (4), based on the addition of complex periodic forcing $k \exp (i \omega t)$ to its first and second equations, so system (4) becomes:

$$
\dot{z}=x+k \exp (i \omega t), \dot{x}=y, \dot{y}=-\alpha y-\beta x-v z-\eta z^{2} \bar{z} \text {. }
$$

(a)

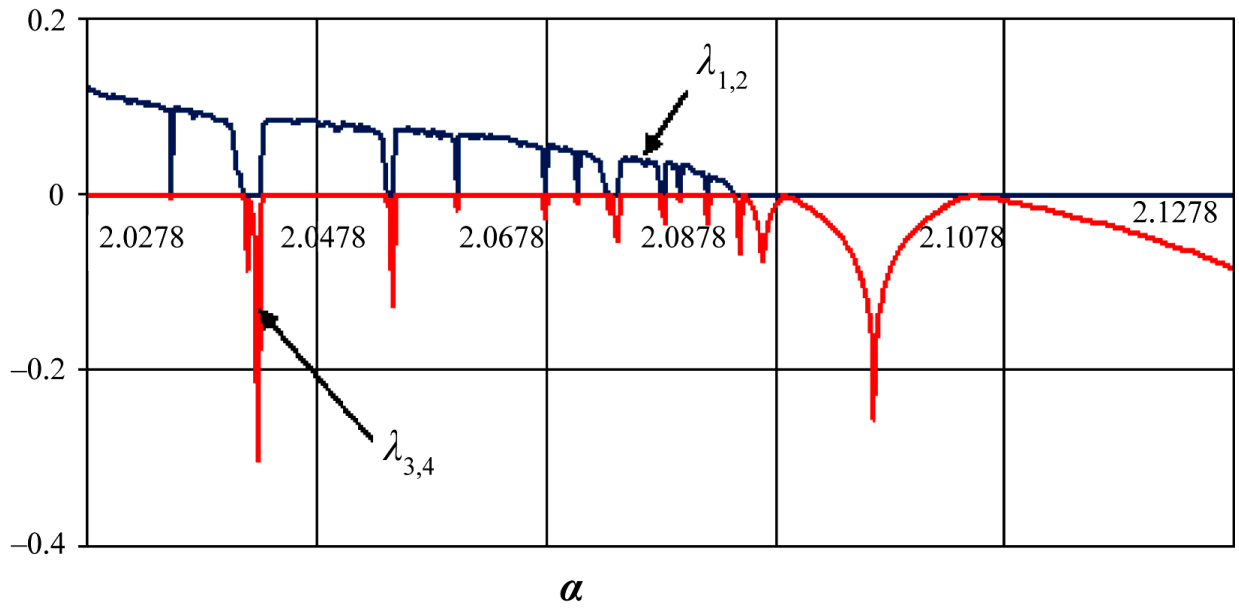

(b)

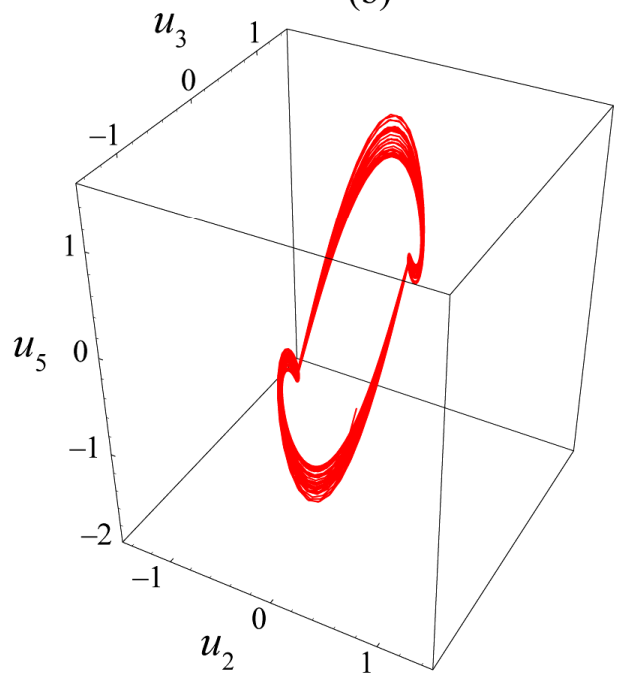

(c)

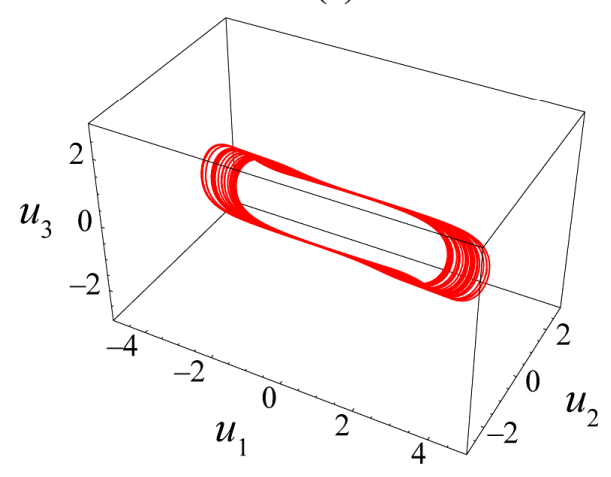

Figure 3. Lyapunov exponents and numerical calculations of the hyperchaotic attractors of (3) using the initial conditions $t_{0}=0, \quad u_{1}(0)=4, \quad u_{2}(0)=0, \quad u_{3}(0)=0, \quad u_{4}(0)=0, \quad u_{5}(0)=-1$ and $u_{6}(0)=0 .(\mathrm{a}) \quad \lambda_{1}, \quad \lambda_{2}, \quad \lambda_{3}$ and $\lambda_{4}$ versus $\alpha ;(\mathrm{b})$ A hyperchaotic attractors of (3) in $\left(u_{2}, u_{3}, u_{5}\right)$ space; (c) A hyperchaotic attractors of (3) in $\left(u_{1}, u_{2}, u_{3}\right)$ space. 
The real version of (15) using $u_{7}=\omega t$ reads:

$$
\begin{aligned}
& \dot{u}_{1}=u_{3}+k \cos \left(u_{6}\right), \\
& \dot{u}_{2}=u_{4}+k \sin \left(u_{6}\right), \\
& \dot{u}_{3}=u_{5}, \dot{u}_{4}=u_{6}, \\
& \dot{u}_{5}=-\alpha u_{5}-\beta u_{3}-v u_{1}-\eta u_{1}\left(u_{1}^{2}+u_{2}^{2}\right), \\
& \dot{u}_{6}=-\alpha u_{6}-\beta u_{4}-v u_{2}-\eta u_{2}\left(u_{1}^{2}+u_{2}^{2}\right), \\
& \dot{u}_{7}=\omega .
\end{aligned}
$$

What we would like to see is whether, by a suitable selection of values of $k$ and $\omega$, one can control the chaotic solutions of system (4) by converting them from chaotic to periodic with frequency $\omega$.

For the choice of $\alpha=1, \beta=4, v=-4.3, \eta=1, \omega=10$ and $k=10$, and the initial conditions $t_{0}=0, u_{1}(0)=4$,

(a)
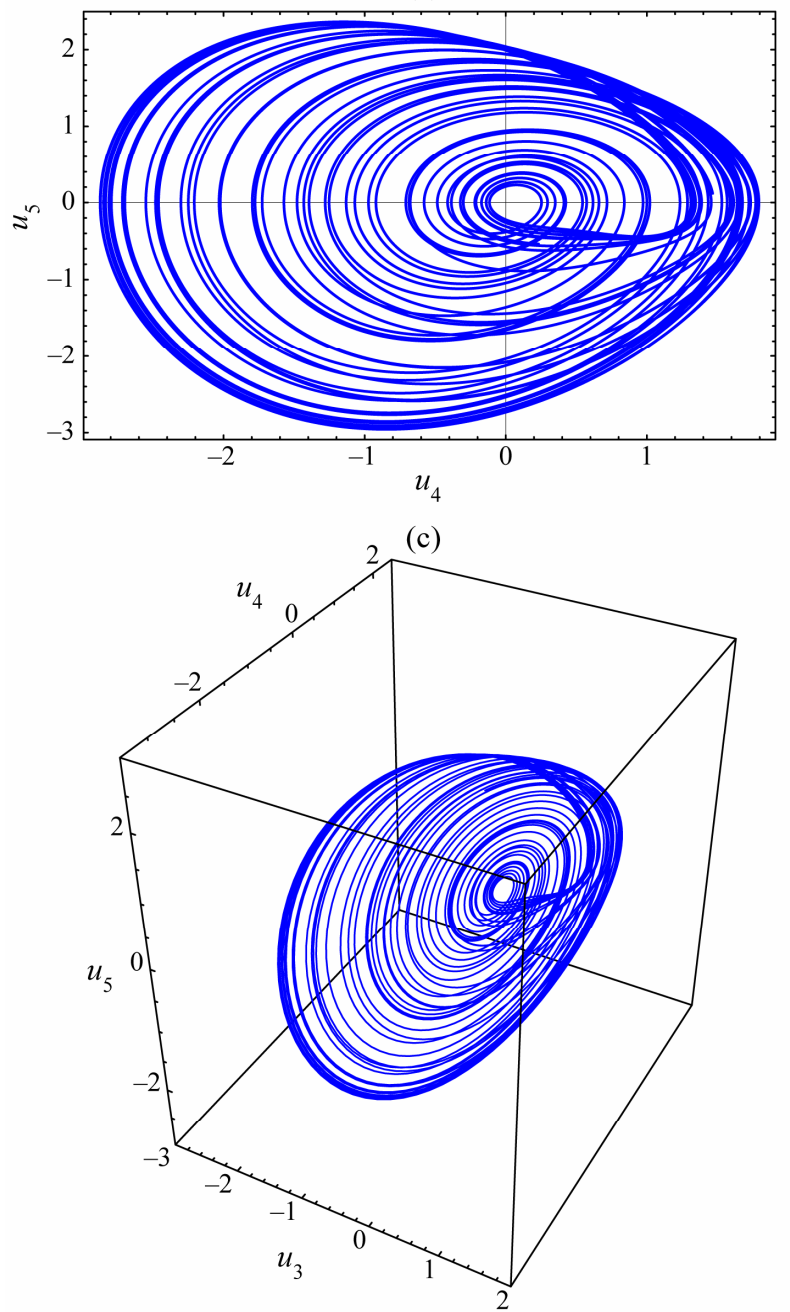

$u_{2}(0)=1, u_{3}(0)=-2, u_{4}(0)=2, u_{5}(0)=-1, u_{6}(0)=1$, $u_{7}(0)=1$, we have the Lyapunov exponents $\lambda_{1}=0$, $\lambda_{2}=-0.02861, \lambda_{3}=-0.02943, \lambda_{4}=-0.72071$, $\lambda_{5}=-0.72155, \lambda_{6}=-1.38533, \lambda_{7}=1$ (for more details about the calculations of Lyapunov exponents, see Ref. [16]. This means that the hyperchaotic attractor of (2) is converted to periodic behavior (see Figures 4(a) and (c) before control and Figures 4(b) and (d) after control).

\section{Conclusions}

In this paper we proposed both chaotic and hyperchaotic complex jerk equations and investigated their dynamics. The stability analysis of the trivial fixed points of these complex equations are studied. These equations appeared in several important applications of physics, engineering, and biology.

(b)
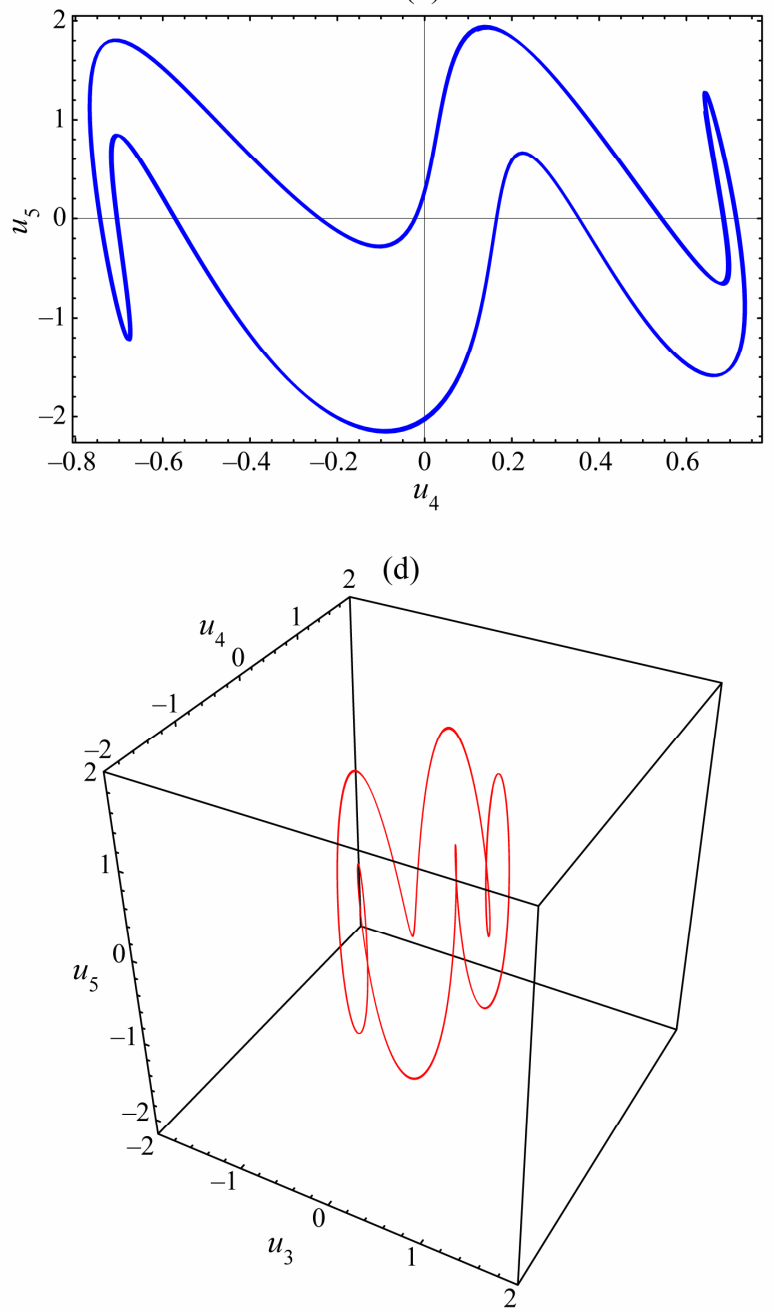

Figure 4. A chaotic attractor of system (15) for $\alpha=1, \beta=4, v=-4.3, \eta=1, k=10, w=10$ and $u_{7}(0)=1$, with the same initial conditions as in Figure 1 and (a) $\left(u_{4}, u_{5}\right)$ plane before control; (b) $\left(u_{4}, u_{5}\right)$ plane after control; (c) $\left(u_{3}, u_{4}, u_{5}\right)$ space before control; (d) $\left(u_{3}, u_{4}, u_{5}\right)$ space after control. 
Both of our examples (2) and (3) are symmetric and dissipative under the condition $\alpha>0$. The chaotic Equation (2) has both isolated and non-isolated fixed points. The projection of non-isolated fixed point in $\left(u_{1}, u_{2}\right)$ space is a circle with center $(0,0)$. The dynamic of system (2) is very complicated as shown in Figure 1, since it has, solutions approach to fixed points, periodic solutions, quasi-periodic solutions and chaotic behavior. The hyperchaotic Equation (3) has only one fixed point. The values of the parameter $\alpha$ at which (3) has hyperchaotic attractors is calculated. The control of chaotic attractors of Equation (2) is studied by adding a complex periodic forcing and the results are shown in Figure 4. Other examples of Equation (1) can be similarly studied and investigated as we did for Equations (2) and (3).

\section{REFERENCES}

[1] E. N. Lorenz, "Deterministic Nonperiodic Flow," Journal of Atmospheric Sciences, Vol. 20, No. 2, 1963, pp. 130141.

doi:10.1175/1520-0469(1963)020<0130:DNF>2.0.CO;2

[2] O. E. Rössler, "An Equation for Continuous chaos," Physics Letters A, Vol. 57, No. 5, 1976, pp. 397-398. doi:10.1016/0375-9601(76)90101-8

[3] O. E. Rossler, "An Equation for Hyperchaos," Physics Letters A, Vol. 71, No. 2-3, 1979, pp. 155-157. doi:10.1016/0375-9601(79)90150-6

[4] J. C. Sprott, "Simple Chaotic Systems and Circuits," American Journal of Physicals, Vol. 68, No. 8, 2000, pp. 758-763. doi:10.1119/1.19538

[5] J. C. Sprott, "Some Simple Chaotic Flows," Physical Review E, Vol. 50, No. 2, 1994, pp. R647-R650. doi:10.1103/PhysRevE.50.R647

[6] H. P. W. Gottlieb, "Question \#38. What Is the Simplest Jerk Function That Gives Chaos?" American Journal of Physicals, Vol. 64, No. 5, 1996, p. 525. doi:10.1119/1.18276

[7] S. J. Linz, "Nonlinear Dynamical Models and Jerk Motion," American Journal of Physicals, Vol. 65, No. 6, 1997, pp. 523-526. doi:10.1119/1.18594

[8] J. C. Sprott, "Some Simple Chaotic Jerk Functions," American Journal of Physicals, Vol. 65, No. 6, 1997, pp. 537-543. doi:10.1119/1.18585

[9] J.-M. Malasoma, "What Is the Simplest Dissipative Chaotic Jerk Equation Which Is Parity Invariant," Physics Letters A, Vol. 264, No. 5, 2000, pp. 383-389. doi:10.1016/S0375-9601(99)00819-1
[10] F. Zhang, J. Heidel and R. L. Borne, "Determining Nonchaotic Parameter Regions in Some Simple Jerk Functions," Chaos, Solitons \& Fractals, Vol. 36, No. 4, 2008, pp. 862-873. doi:10.1016/j.chaos.2006.07.005

[11] R. Eichhorn, S. J. Linz and P. Hänggi, "Simple Polynomial Classes of Chaotic Jerk Dynamics," Chaos, Solitons \& Fractals, Vol. 13, No. 1, 2002, pp. 1-15. doi:10.1016/S0960-0779(00)00237-X

[12] S. J. Linz, "Non-Chaos Criteria for Certain Jerk Dynamics," Physics Letters A, Vol. 275, No. 3, 2000, pp. 204210. doi:10.1016/S0375-9601(00)00576-4

[13] J. C. Sprott, "Chaos and Time-Series Analysis," Oxford University Press, New York, 2003.

[14] L. M. Kocić and S. Gegovska-Zajkova, "On a Jerk Dynamical System," Automatic Control and Robotics, Vol. 8, 2009, pp. 35-44.

[15] G. M. Mahmoud and T. Bountis, "The Dynamics of Systems of Complex Nonlinear Oscillators," International Journal of Bifurcation and Chaos, Vol. 14, No. 1, 2004, pp. 3821-3846. doi:10.1142/S0218127404011624

[16] G. M. Mahmoud, M. E. Ahmed and E. E. Mahmoud, "Analysis of Hyperchaotic Complex Lorenz Systems," International Journal of Modern Physics C, Vol. 19, No. 10, 2008, pp. 1477-1494. doi:10.1142/S0129183108013151

[17] G. M. Mahmoud, M. A. Al-Kashif and A. A. Farghaly, "Chaotic and Hyperchaotic Attractors of a Complex Nonlinear System" Journal of Physicss A: Mathematical and Theoretical, Vol. 41, No. 5, 2008, pp. 55-104. doi:10.1088/1751-8113/41/5/055104

[18] G. M. Mahmoud, E. E. Mahmoud and M. E. Ahmed, "On the Hyperchaotic Complex Lü System," Nonlinear Dynamics, Vol. 58, No. 4, 2008, pp. 725-738. doi:10.1007/s11071-009-9513-0

[19] G. M. Mahmoud, T. Bountis, M. A. Al-Kashif and S. A. Aly, "Dynamical properties and synchronization of complex non-linear equations for detuned laser," J. Dynamical Systems: An International Journal, Vol. 24, No. 1, 2009, pp. 63-79. doi:10.1080/14689360802438298

[20] G. M. Mahmoud and E. E. Mahmoud, "Synchronization and Control of Hyperchaotic Complex Lorenz System," Mathematics and Computers in Simulation, Vol. 80, No. 12, 2010, pp. 2286-2296. doi:10.1016/j.matcom.2010.03.012

[21] G. M. Mahmoud, and E. E. Mahmoud, "Complete Synchronization of Chaotic Complex Nonlinear Systems with Uncertain Parameters," Nonlinear Dynamics, Vol. 62, No. 4, 2010, pp. 875-882. doi:10.1007/s11071-010-9770-y 